\title{
Electrophysiological Effects of Repeated Administration of Agomelatine on the Dopamine, Norepinephrine, and Serotonin Systems in the Rat Brain
}

\author{
Franck Chenu', Mostafa EI Mansari' and Pierre Blier*,, 1,2 \\ 'Institute of Mental Health Research, University of Ottawa, Ottawa, ON, Canada; ${ }^{2}$ Department of Cellular and Molecular Medicine, \\ University of Ottawa, Ottawa, ON, Canada
}

\begin{abstract}
Agomelatine is a melatonergic MTI/MT2 agonist and a serotonin (5-HT) 5- $\mathrm{HT}_{2 \mathrm{C}}$ antagonist. The effects of 2-day and I4-day administration of agomelatine were investigated on the activity of ventral tegmental area (VTA) dopamine (DA), locus coeruleus (LC) norepinephrine (NE), and dorsal raphe nucleus (DRN) 5-HT neurons using in vivo electrophysiology in rats. The 5-HT IA transmission was assessed at hippocampus CA3 pyramidal neurons. After a 2-day regimen of agomelatine $(40 \mathrm{mg} / \mathrm{kg} /$ day, i.p.), an increase in the number of spontaneously active VTA-DA neurons $(p<0.00 I)$ and in the firing rate of LC-NE neurons $(p<0.00 \mathrm{I})$ was observed. After I4 days, the administration of agomelatine induced an increase in: $(1)$ the number of spontaneously active DA neurons $(p<0.05),(2)$ the bursting activity of DA neurons (bursts/min, $p<0.01$ and percentage of spikes occurring in bursts, $p<0.05$ ), (3) the firing rate of DRN-5-HT neurons $(p<0.05)$, and (4) the tonic activation of postsynaptic 5-HT IA receptors located in the hippocampus. The increase in 5-HT firing rate was D2 dependent, as it was antagonized by the D2 receptor antagonist paliperidone. The enhancement of NE firing was restored by the $5-\mathrm{HT}_{2 \mathrm{~A}}$ receptor antagonist MDL-100,907 after the 14-day regimen. All the effects of agomelatine were antagonized by a single administration of the melatonergic antagonist S22153 (except for the increase in the percentage of spikes occurring in burst for DA neurons). The present results suggest that (I) agomelatine exerts direct (2 days) and indirect (I 4 days) modulations of monoaminergic neuronal activity and (2) the melatonergic agonistic activity of agomelatine contributes to the enhancement of DA and 5-HT neurotransmission.
\end{abstract}

Neuropsychopharmacology (20I3) 38, 275-284; doi:I0.1038/npp.20 I2.140; published online 8 August 2012

Keywords: agomelatine; antidepressant; electrophysiology; dopamine; norepinephrine; serotonin

\section{INTRODUCTION}

Agomelatine is an antidepressant with a novel mechanism of action. It is a melatonergic agonist for MT1 and MT2 receptors and a serotonin $(5-\mathrm{HT})_{2 \mathrm{C}}$ receptor antagonist (Millan et al, 2003; Audinot et al, 2003; de Bodinat et al, 2010; Kasper and Hamon, 2009; Ying et al, 1996). The antidepressant-like effect of agomelatine has been demonstrated in different animal models. In the forced swimming test, acute and repeated administration of agomelatine induces an antidepressant-like effect in rats (Bourin et al, 2004), whereas in mice only the long-term administration induces a significant increase in swimming duration. In the chronic mild stress (CMS), repeated administration of agomelatine in the evening dose-dependently reverses the

*Correspondence: Dr P Blier, Institute of Mental Health Research (IMHR), II45 Carling Avenue, University of Ottawa, Room 64I2, Ottawa KIZ 7K4, ON, Canada, Tel: + I 6137226521 (ext 6944), Fax: + | 6137613610, E-mail: pierre.blier@theroyal.ca

Received 16 March 2012; revised 26 June 20I2; accepted 28 June 2012
CMS-induced reduction in sucrose consumption, suggesting a reduction of anhedonia in stressed animals (Papp et al, 2003). This effect is, at least in part, related to the melatonergic component of the drug as it is blocked by a single administration of the MT1/MT2 receptor antagonist S22153. In the learned helplessness (LH) model, agomelatine induces an antidepressant-like activity after chronic administration, which was abolished by pretreatment with the MT1/MT2 receptor antagonist S22153 (Bertaina-Anglade et al, 2006), suggesting the involvement of the melatonergic receptors. These behavioral data results are supported by studies showing that the administration of the melatonergic receptor antagonist S22153 antagonized the increase in brain-derived neurotrophic factor (BDNF) mRNA levels in rat prefrontal cortex (Racagni et al, 2011).

As both the $5-\mathrm{HT}_{2 \mathrm{C}}$ receptor antagonist SB-242084 and melatonin are devoid of effect by themselves in the CMS and $\mathrm{LH}$, these preclinical data suggest that the antidepressantlike effect of agomelatine requires both the activation of melatonergic receptors and the blockade of $5-\mathrm{HT}_{2 \mathrm{C}}$ receptors. This is in line with clinical studies showing that 
melatonin by itself does not have any antidepressant effect in patients suffering from major depressive disorder (MDD; Dalton et al, 2000; Dolberg et al, 1998). In contrast, the efficacy of agomelatine in MDD has been demonstrated in clinical trials $v s$ placebo (Kennedy and Emsley, 2006) and using active comparators such as venlafaxine (Lemoine et al, 2007), sertraline (Kasper et al, 2010), and fluoxetine (Hale et al, 2010).

Regarding its putative mechanism of action, agomelatine induces a dose-dependent enhancement of extracellular frontocortical dopamine (DA) and norepinephrine (NE) transmission measured by microdialysis (Millan et al, 2003). These increases are unaffected by the melatonin receptor antagonist S22153 and cannot be mimicked by melatonin. However, previous results showed that $5-\mathrm{HT}_{2 \mathrm{C}}$ receptor antagonists induce an increase in extracellular levels of NE and DA in the prefrontal cortex of freely moving rats (Gobert et al, 2000). Therefore, this effect is likely associated with the $5-\mathrm{HT}_{2 \mathrm{C}}$ receptor antagonist property of agomelatine. A dose-dependent increase in extracellular levels of NE was also found in the dorsal hippocampus of freely moving rats following an acute administration of agomelatine (Millan et al, 2005). These findings are supported by electrophysiology results showing a dose-dependent increase in the firing rate of locus coeruleus (LC) NE neurons (Millan et al, 2003) following acute administration of incremental doses of agomelatine. Unexpectedly, the electrical activity of ventral tegmental area (VTA) DA neurons was unchanged.

Based on all these previous preclinical studies, it seems that there is a discrepancy between the behavioral and the neurobiochemical effects of agomelatine. Indeed, the antidepressant-like effect of agomelatine cannot be fully explained by the increase in extracellular levels of $\mathrm{NE}$ and DA because this increase is not antagonized by the melatonergic receptor antagonist S22153, whereas its antidepressant-like effect is. The aim of this study was to investigate the effects of short (2 days) and long-term (14 days) administration of agomelatine on the electrical activity of VTA-DA neurons, LC-NE neurons, and dorsal raphe nuclei (DRN) 5-HT neurons in order to have a better understanding of its mechanism of action.

\section{MATERIALS AND METHODS}

\section{Animals}

Male Sprague-Dawley rats (Charles River, St Constant, QC, Canada) weighing 250 to $330 \mathrm{~g}$, were used for the experiments. They were kept under standard laboratory conditions $(12: 12 \mathrm{~h}$ light/dark cycle, free access to food and water). All animals were handled according to the guidelines of the Canadian Council on Animal Care. Protocols in this study were approved by the local Animal Care Committee (IMHR, Ottawa, ON, Canada).

\section{Chemical Compounds}

Agomelatine and S22153 (N-[2-(5-ethyl-benzo[b]thien-3yl)ethyl]) acetamide were provided by Servier and they were suspended in hydroxyethylcellulose (HEC) $1 \%$. Agomelatine ( $40 \mathrm{mg} / \mathrm{kg} /$ day) was injected intraperitoneally (i.p.) daily at
$1700 \mathrm{~h}$. 5-HT, quisqualic acid, and WAY-100,635 were bought from Sigma (Oakville, ON, Canada). Paliperidone was provided by Janssen (Titusville, NJ).

\section{In Vivo Electrophysiological Recordings}

Rats were anesthetized with chloral hydrate ( $400 \mathrm{mg} / \mathrm{kg}$, i.p.) and placed into a stereotaxic frame. The extracellular recordings of 5-HT, DA, and NE neurons in the DR, the VTA, and the LC, respectively, were carried out using single-barrelled glass micropipettes (Stoelting, Spencerville, MD) preloaded with a $2 \mathrm{M} \mathrm{NaCl}$ solution (impedance 4$7 \mathrm{M} \Omega$ ). The extracellular recordings of pyramidal neurons in the $\mathrm{CA}_{3}$ region of the hippocampus were carried out using multi-barrelled glass micropipettes (impedances: central barrel: $2-5 \mathrm{M} \Omega$, side barrels: $20-30 \mathrm{M} \Omega$ ). The central barrel used for extracellular unitary recording and one side barrel (automatic current balancing) were filled with $2 \mathrm{M} \mathrm{NaCl}$ solution. The three other side barrels were filled with 5-HT $(25 \mathrm{mM}$ in $0.2 \mathrm{M} \mathrm{NaCl}, \mathrm{pH}=4)$ and quisqualate $(1.5 \mathrm{mM}$ in $0.2 \mathrm{M} \mathrm{NaCl} ; \mathrm{pH}=8$ ). 5-HT was ejected as cations and retained with currents of -10 to $-8 \mathrm{nA}$. Quisqualate was ejected as an anion and retained with a current of $+5 \mathrm{nA}$.

\section{Recording of DRN-5-HT Neurons}

The single-barrelled glass micropipettes were positioned using the following coordinates (in $\mathrm{mm}$ from $\lambda$ ): AP: +1.0 to 1.2 ; L: $0 \pm 0.1$; and V: 5 to 7 . The presumed 5-HT neurons were then identified using the following criteria: a slow (0.5$2.5 \mathrm{~Hz})$ and regular firing rate and long-duration $(2-5 \mathrm{~ms})$ bi- or tri-phasic extracellular waveform (Aghajanian and Vandermaelen, 1982). It is important to mention that there are slow firing neurons in the DRN that are not serotonergic (Allers and Sharp, 2003). However, these neurons have shorter-duration spikes and do not fire as regularly as $5-\mathrm{HT}$ neurons. It was deemed that few, if any, such neurons were accidentally introduced in our tally of 5-HT neurons by adhering to the above-mentioned criteria. As previously demonstrated, some 5-HT neurons display a bursting activity (Hajos et al, 1995). This occasional firing pattern of 5-HT neurons was analyzed by spike interval burst analysis. The onset of a burst was defined as the occurrence of two spikes with an interspike interval (ISI) of $<10 \mathrm{~ms}$.

\section{Recording of VTA-DA Neurons}

The single-barrelled glass micropipettes were positioned using the following coordinates (in $\mathrm{mm}$ from bregma): AP: -6 to -5.4 ; L: 0.6 to 1 ; and V: 7 to 9 . The presumed DA neurons were identified according to well-established electrophysiological properties in vivo: a typical triphasic action potential with a marked negative deflection, a characteristic long duration $(>2.5 \mathrm{~ms})$ often with an inflection or 'notch' on the rising phase, and a slow spontaneous firing rate $(2-10 \mathrm{~Hz})$ with an irregular single spiking pattern with slow bursting activity characterized by spike-amplitude decrement (Grace and Bunney, 1983). As previously described, a criterion of duration $(>1.1 \mathrm{~ms}$ from the start of the action potential to the negative trough) was also used (Ungless et al, 2004). The firing pattern of recorded DA neurons was analyzed by ISI burst analysis 
following the criteria established by Grace and Bunney (1984). The onset of a burst was defined as the occurrence of two spikes with an ISI of $<0.08 \mathrm{~s}$. The termination of bursts was defined as an ISI of $\geqslant 0.16 \mathrm{~s}$. Bursts parameters studied were: number of bursts/min, percentage of spikes occurring in bursts, number of spikes/burst, and ISI within bursts. To define the number of DA neurons per track, we used the technique previously described by Valenti et al (2011). Briefly, 9 tracks were performed on a grid of $600 \mu \mathrm{m} \times 600 \mu \mathrm{m}$ : AP: 3.2 to $3.6 \mathrm{~mm}, \mathrm{~L}: 0.6$ to $1 \mathrm{~mm}$ ( 3 tracks per row, each track separated by $200 \mu \mathrm{m}$ ).

\section{Recording of LC-NE Neurons}

The single-barrelled glass micropipettes were positioned using the following coordinates (in $\mathrm{mm}$ from $\lambda$ ): AP: -1.0 to -1.2 ; L: 1.0 to 1.3 ; and V: 5 to 7 . Spontaneously active NE neurons were identified using the following criteria: regular firing rate $(0.5-5.0 \mathrm{~Hz})$ and positive action potential of long duration (0.8-1.2 $\mathrm{ms})$ exhibiting a brisk excitatory response to a nociceptive pinch of the contralateral hind paw (Aghajanian and Vandermaelen, 1982). The compression lasted $\sim 1 \mathrm{~s}$ with equal pressure being applied to the paw of rats with forceps.

\section{Recording of Pyramidal Neurons of the $\mathrm{CA}_{3}$ Region of the Dorsal Hippocampus}

The five-barrelled glass micropipettes were positioned using the following coordinates (in mm from $\lambda$ ): AP: 3.8 to 4.5 ; L: 4 to 4.2 ; and $\mathrm{V}: 3$ to 4.5 . Stimulated quisqualate pyramidal neurons were identified by their high amplitude (0.5$1.2 \mathrm{mV})$, high frequency $(8-12 \mathrm{~Hz})$, and long duration $(0.6-$ $1.0 \mathrm{~ms}$ ) action potential and by their characteristic 'complex spike' discharge (Kandel et al, 1961). A microiontophoretic application of 5 -HT was performed to determine the $\mathrm{RT}_{50}$ value (s), which represents the time required for the firing activity to recover by $50 \%$ from the cessation of the microiontophoretic application. In this study, the $\mathrm{RT}_{50}$ value was used as an index of the capacity of 5-HT terminals in the dorsal hippocampus to remove 5-HT from the synaptic cleft through its 5-HT reuptake transporters (Pineyro et al, 1994).

\section{Statistical Analyses}

Electrophysiological data are expressed as means \pm SEM of the different parameters. Data were analyzed using a $t$-test or a one-way analysis of variance (ANOVA) for repeated measures, with rat and treatment as main factors. Fisher's protected least significant difference test (Fisher's PLSD test) was used to analyze the statistical significance between groups. A level of $p<0.05$ was accepted as evidence for a statistically significant effect.

\section{RESULTS}

Effect of 2-Day Administration of Agomelatine on DA, NE, and 5-HT Neurons

Injection of agomelatine for 2 days modified neither the firing rate (Figure 1a) nor the pattern of discharge (percent of spikes occurring in bursts: $16 \pm 3$ and $14 \pm 2$; bursts/min: $12 \pm 3$ and $11 \pm 2$ for control and agomelatine-treated rats, respectively; $p>0.05)$ of VTA-DA neurons. However, the number of spontaneously active cells (neurons per track) was significantly increased by $70 \%$ in rats administered with agomelatine in comparison with control rats ( $t$-test, $p<0.001$; Figure $1 \mathrm{a}$ ).

Administration of agomelatine for 2 days induced a $50 \%$ increase in the firing rate of LC-NE neurons in comparison with controls ( $t$-test, $p<0.001$; Figure $1 \mathrm{~b}$ ).

Administration of agomelatine for 2 days did not modify the firing rate or pattern of discharge of DRN-5-HT neurons (Figure 1c).

Effects of the MT1/MT2 Receptor Antagonist S22153 in
Rats Treated with Agomelatine for 2 Days

Rats were given agomelatine for 2 days (daily injection). On day 3, several neurons were first recorded in the VTA (4-5 tracks) or in the LC (2-3 tracks). Then, S22153 (20 mg/kg i.p.) was injected and $20 \mathrm{~min}$ later additional neurons were recorded again from both the VTA (4-5 tracks) and LC (2-3 tracks). In both areas, S22153 was devoid of effect in control animals (VTA: cells per track $1.4 \pm 0.2$ and $1.3 \pm 0.2$, $p>0.05$; LC: $1.8 \pm 0.1$ and $2.0 \pm 0.2, p>0.05$; before and after S22153, respectively).

The increase in the number of DA neurons recorded per track in the VTA after a 2-day regimen of agomelatine was antagonized by a single administration of S22153 at $20 \mathrm{~min}$ before the recording (one-way ANOVA for repeated measure: $F_{1,6}=23.1 ; p<0.01$; Figure $1 d$ ). This suggests that this enhancement was, at least in part, mediated by the melatonergic component of agomelatine.

A single dose of S22153 also antagonized the increase in LC-NE firing rate induced by administration of agomelatine (one-way ANOVA for repeated measure: $\mathrm{F}_{1,7}=13.1$; $p<0.01$; Figure 1e). This suggests that this increase in firing rate was, at least in part, mediated by the melatonergic component of agomelatine.

\section{Effects of 14-Day Administration of Agomelatine on VTA-DA Neurons}

After long-term administration of agomelatine, the firing rate of DA neurons remained unchanged in treated rats in comparison with control (Figure 2a), whereas the number of spontaneously active neurons (cells per track) was still significantly increased by $60 \%$ (Figure 2a). Their pattern of discharge was, however, modified as there was a significant increase in bursting activity. Both the number of bursts per min and the percentage of spikes occurring in burst were significantly increased by $90 \% \quad(p<0.01$ and $p<0.05$, respectively; Figure 2a).

\section{Effect of 14-Day Administration of Agomelatine on DRN-5-HT Neurons}

After long-term administration of agomelatine, the firing rate of DRN-5-HT neurons was significantly increased by $40 \%(p<0.05$; Figure $2 \mathrm{~b})$, whereas it was not after a 2 -day treatment. As the 2-day regimen left unaltered the firing rate of 5-HT neurons, the latter results suggested that this was an indirect effect of agomelatine. As DA firing activity was increased after 14 days of agomelatine, and that DA has an excitatory effect on 5-HT firing through the activation of 
a Dopamine Neurons

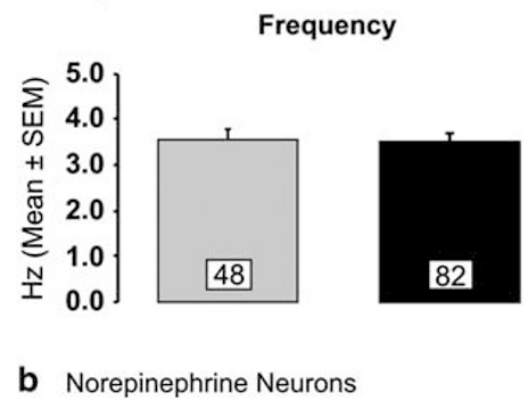

b Norepinephrine Neurons

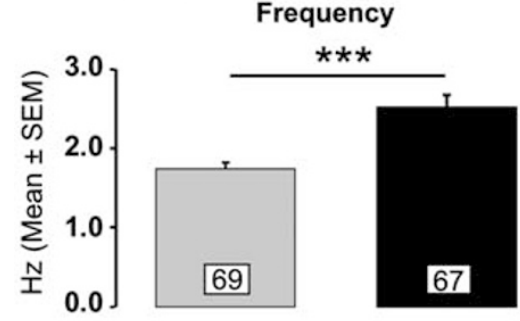

d Dopamine Neurons

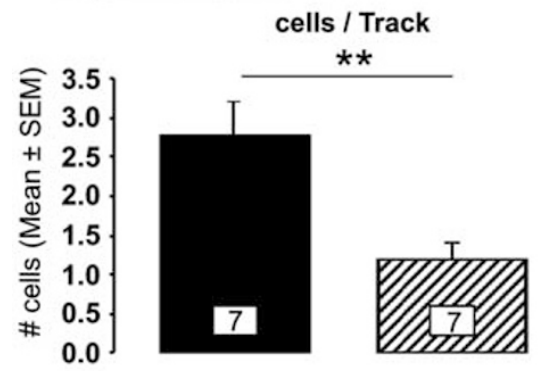

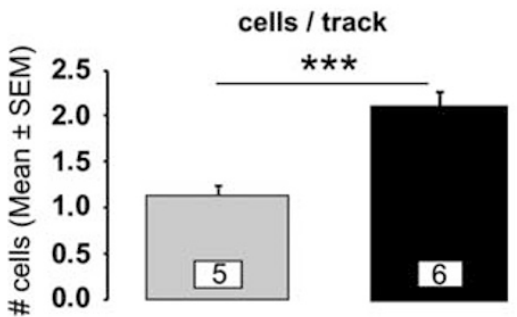

C Serotonin Neurons

Frequency

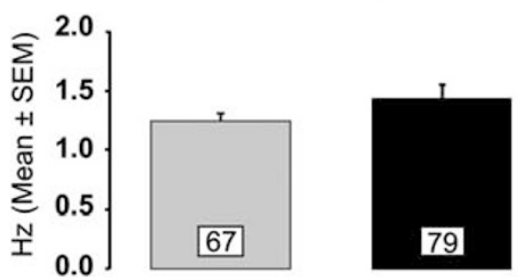

e Norepinephrine Neurons

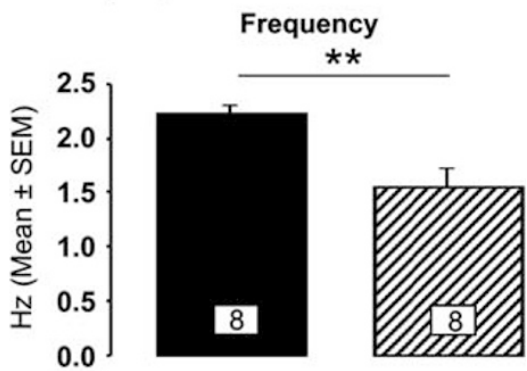

\section{Control $\square$ Agomelatine ( $\times 2$ days)}

Agomelatine ( $x 2$ days $)+S 22153$

Figure I (a-c) Effect of agomelatine administration (2 days) on the firing activity of VTA-DA, LC-NE, and DRN-5-HT neurons. (d, e) Effect of melatonergic blockade with S22153 on the excitatory effect of agomelatine on the number of spontaneously DA active cells found per track in the VTA and on the firing rate of NE neurons in the LC. Data are expressed as means \pm SEM of the firing rate and the number of spontaneously active cells found per track (DA neurons only). The number of neurons (firing rate) or rats (cells per track) recorded in each group is provided within the histograms. (a-c) Data were analyzed using t-test. (d, e) Data were analyzed by one-way ANOVA for repeated measures followed by Fisher's PLSD test. *Significant effect of the treatment (agomelatine or S22153) in comparison with the corresponding control group, $* * * 0.01$, $* * * * 0.001$.

DA D2 receptors (Aman et al, 2007; Haj-Dahmane, 2001), it was postulated that the increase in 5-HT neurotransmission might be a consequence of an enhanced DA transmission. In order to test this hypothesis, several 5-HT neurons were recorded before and after the injection of the D2 antagonist paliperidone $(1 \mathrm{mg} / \mathrm{kg}$, i.v. $)$ in the same rats. Similar to previously published data (Chernoloz et al, 2009; Dremencov et al, 2007a), paliperidone was devoid of effect in control rats $(1.0 \pm 0.1$ and $0.9 \pm 0.2 \mathrm{~Hz}$ before and after paliperidone, respectively; $p>0.05$ ) but antagonized the increase in firing rate in agomelatine-treated rats $\left(\mathrm{F}_{1,4}=35.0 ; p<0.01\right.$; Figure $\left.2 \mathrm{~b}\right)$.

\section{Effect of 14-Day Administration of Agomelatine on LC-NE Neurons}

After long-term administration of agomelatine, the firing rate of LC-NE neurons was back to normal when compared with controls (Figure 2c). As it was previously demonstrated that an increase in 5-HT transmission following the administration of SSRIs induces an activation of $5-\mathrm{HT}_{2 \mathrm{~A}}$ receptors located on GABA interneurons that in turn decrease the firing rate of LC-NE neurons (Szabo and Blier, 2001), it was hypothesized that an increase in 5-HT transmission might account for the reversal to normal of NE firing rate. To confirm this assumption, NE neurons were recorded before and after the administration of the $5-\mathrm{HT}_{2 \mathrm{~A}}$ receptor antagonist MDL-100,907 (0.2 mg/kg, i.v.) in the same rats. Similar to previously published data (Szabo and Blier, 2001), MDL-100,907 was devoid of effect in control rats $(1.8 \pm 0.2$ and $1.7 \pm 0.3 \mathrm{~Hz}$ before and after MDL-100,907, respectively; $p>0.05$ ) but increased the firing rate of $\mathrm{NE}$ neurons in 14-day agomelatine-treated rats by $50 \%$ (oneway ANOVA for repeated measures: $\mathrm{F}_{1,2}=70.3 ; p<0.05$; Figure 2c). 
a Dopamine Neurons

Frequency
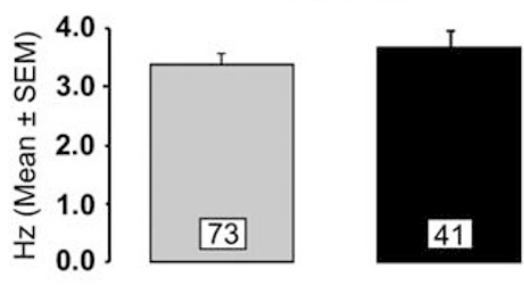

Cells / track

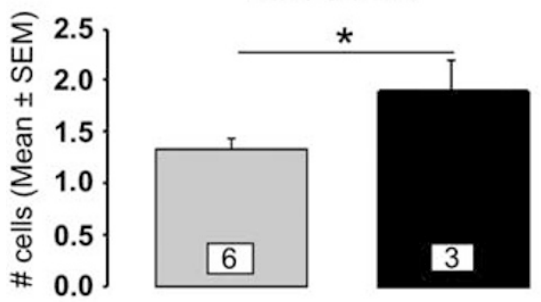

b Serotonin Neurons

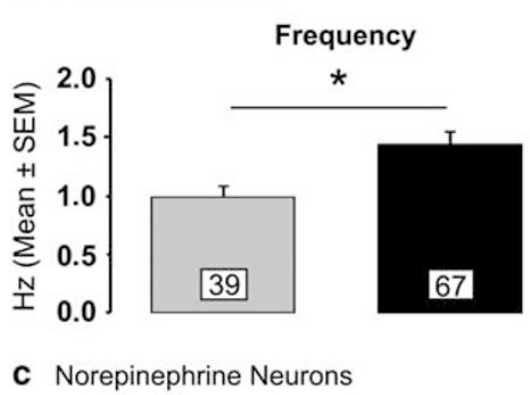

Frequency
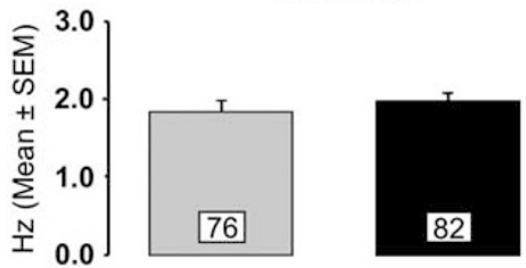
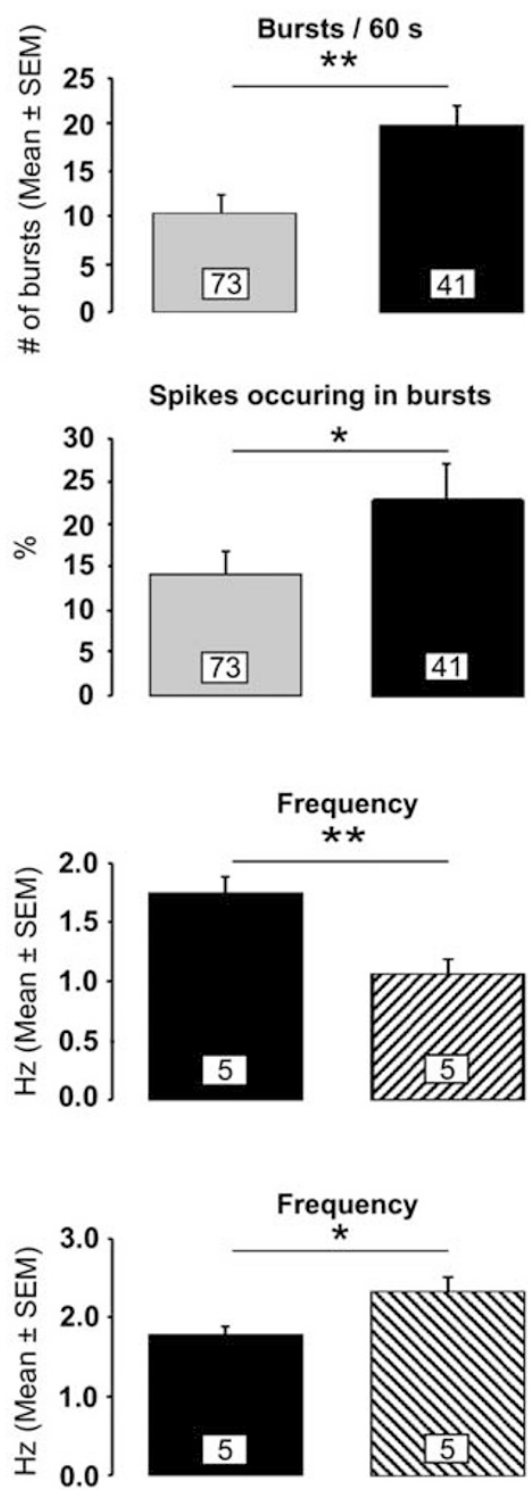

Spikes occuring in bursts
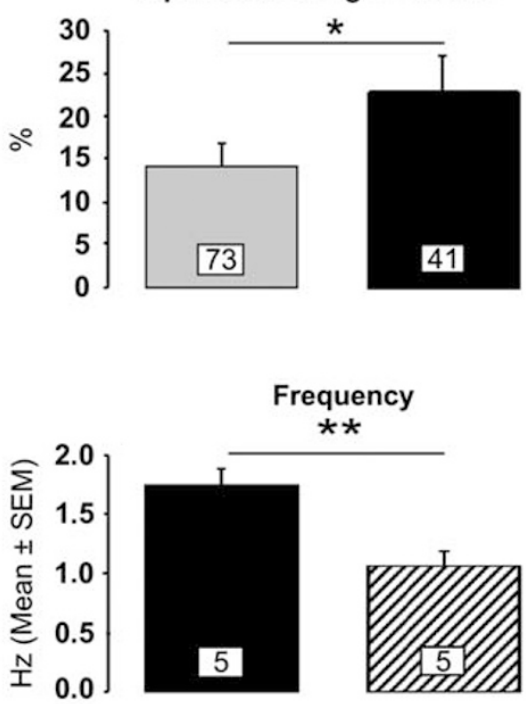

Control

Agomelatine (x 14 days)

Figure 2 Effect of agomelatine ( $\mathbf{0}$ ) administration (I 4 days) on the firing activity of (a) VTA-DA neurons, (b) DRN-5-HT neurons, and (c) LC-NE neurons in comparison with control ( $)$. Data are expressed as means \pm SEM of the firing rate, the number of spontaneously active cells found per track, the number of bursts/min, and the percentage of spikes occurring in burst. The number of neurons or rats recorded in each group is provided within the histograms. Data were analyzed using t-test or one-way ANOVA for repeated measures followed by Fisher's PLSD test (paliperidone and MDL- I00,907). *Significant effect of the treatment (agomelatine, paliperidone, or MDL-100,907) in comparison with the corresponding control group, * $p<0.05$, ** $p<0.01$.

Effects of the MT1/MT2 Antagonist S22153 in Rats Treated with Agomelatine for 14 Days

Rats were given agomelatine for 14 days. On day 15, several neurons were recorded in the VTA (4-5 tracks) or in the DRN (2-3 tracks). Then, S22153 ( $20 \mathrm{mg} / \mathrm{kg}$, i.p.) was injected and $20 \mathrm{~min}$ later more neurons were recorded in both the VTA (4-5 tracks) and DRN (2-3 tracks). S22153 was devoid of effect in control animals in both the VTA (firing rate: $4.4 \pm 0.3$ and $4.1 \pm 0.2, p>0.05$; bursts/min: $15 \pm 3$ and $13 \pm 3, p>0.05$; percent of spikes in bursts: $18 \pm 4$ and $15 \pm 3, p>0.05$; before and after administration of S22153, respectively) and the DRN (1.1 \pm 0.1 and $1.3 \pm 0.2$. before and after administration of S22153, respectively; $p>0.05)$.

Similar to what was observed after the 2-day regimen of agomelatine, S22153 induced a significant decrease in the number of spontaneously active DA neurons (one-way ANOVA for repeated measures: $\mathrm{F}_{1,9}=11.4 ; p<0.01$; Figure 3a). The percentage of spikes occurring in bursts was not significantly decreased $(p=0.052)$. Nonetheless, both the number of bursts per min (one-way ANOVA for 
a Dopamine Neurons
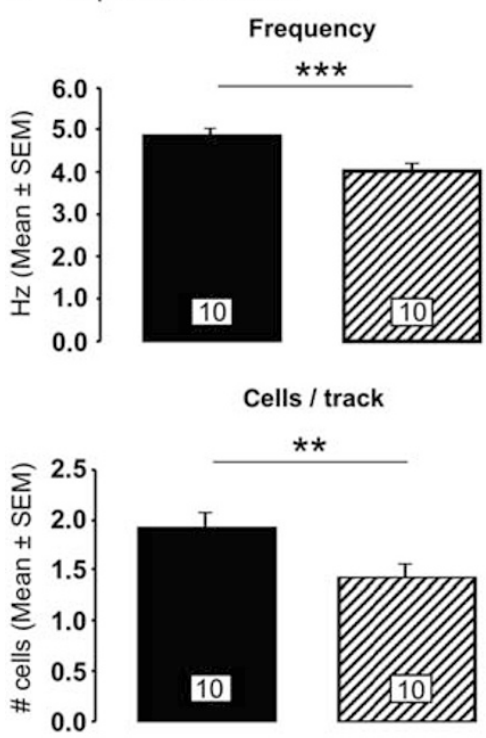

b Serotonin Neurons

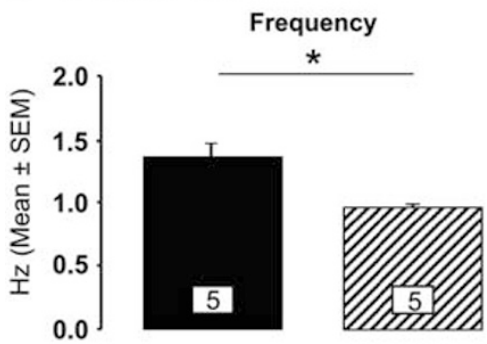

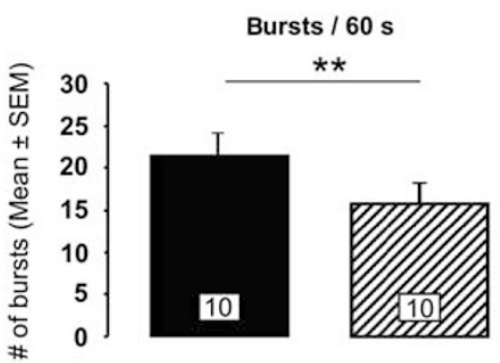

Spikes occuring in bursts

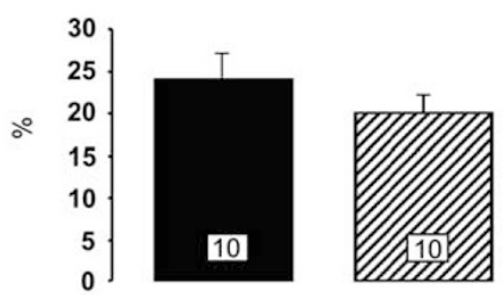

Agomelatine (x 14 days)

Agomelatine (x 14 days) + S22153

Figure 3 Effect of melatonergic blockade with S22I53 on the activity of agomelatine ( 14 days) on (a) the firing pattern of VTA-DA neurons and on (b) the firing rate of 5-HT neurons in the DRN. The number of animals recorded in each group is provided within the histograms. Data were analyzed by one-way ANOVA for repeated measures followed by Fisher's PLSD test. *Significant effect of S22I53 (hatched) in comparison with the corresponding I4-day agomelatine-treated group $(\mathbf{\square}), * p<0.05$, $* * * 0.0$ I, $* * * * p<0.00$ I.

repeated measures: $\mathrm{F}_{1,9}=14 ; p<0.01$; Figure $3 \mathrm{a}$ ) and the firing rate (one-way ANOVA for repeated measures: $\mathrm{F}_{1,9}=24.3 ; p<0.001$; Figure $3 \mathrm{a}$ ) were significantly decreased, indicating a restoration of normal firing activity of VTA-DA neurons by the melatonin antagonist S22153.

A single administration of $\mathrm{S} 22153$ reversed the increase in 5-HT firing rate induced by a prolonged administration of agomelatine (one-way ANOVA for repeated measures: $\mathrm{F}_{1,4}=15.1 ; p<0.05$; Figure $\left.3 \mathrm{~b}\right)$. This observation is in line with the return to normal of DA firing activity observed in the VTA in agomelatine-treated rats following the injection of S22153.

\section{Effect of Agomelatine on 5-HT ${ }_{1 \mathrm{~A}}$ Transmission in the Hippocampus}

As expected, there was no change in the $\mathrm{IT}_{50}$ index and $\mathrm{RT}_{50}$ duration, suggesting that there was no change in sensitivity of the $5-\mathrm{HT}_{1 \mathrm{~A}}$ receptors and no changes in the reuptake of 5-HT, respectively (Figure 4).

The intravenous administration of incremental doses of the selective $5-\mathrm{HT}_{1 \mathrm{~A}}$ receptor antagonist WAY-100,635 $(25-100 \mu \mathrm{g} / \mathrm{kg})$ did not modify the firing activity of dorsal hippocampus $\mathrm{CA}_{3}$ pyramidal neurons in control rats, as previously reported (Haddjeri et al, 1998). In contrast, in the rats treated with agomelatine for a 2-week period, the administration of WAY-100,635 significantly increased the firing activity of $\mathrm{CA}_{3}$ pyramidal neurons $\left(F_{1,64}=21.3\right.$, $p<0.001$; Figure 4), therefore indicating a greater tonic activation of the inhibitory postsynaptic $5-\mathrm{HT}_{1 \mathrm{~A}}$ receptors.

\section{DISCUSSION}

The present results suggest that agomelatine exerts direct and indirect modulation of monoaminergic neuronal activity. Indeed, after a 2-day regimen, agomelatine (1) enhanced VTA-DA activity (number of spontaneously active neurons), (2) enhanced LC-NE firing rate, but (3) had no effect on DRN-5-HT firing activity. After a 14-day regimen, (1) the VTA-DA activity was enhanced (spontaneously active neurons and burst activity), (2) DRN-5-HT firing rate was increased through a D2-dependent mechanism, and (3) LC-NE firing activity was dampened back to normal because of the enhanced negative feedback exerted by upregulated 5-HT neurotransmission.

The present findings obtained after a 2-day regimen of agomelatine support previous results showing that its acute administration induces an increase in the firing rate of 
a

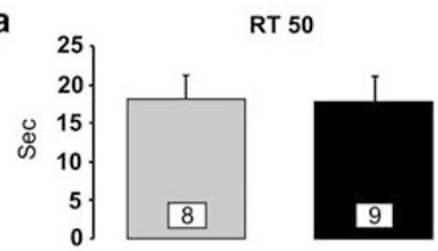

b

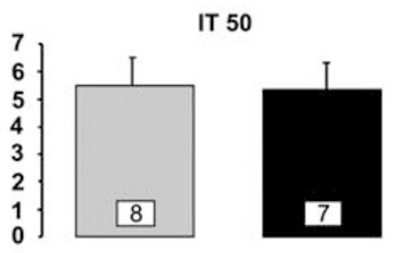

C

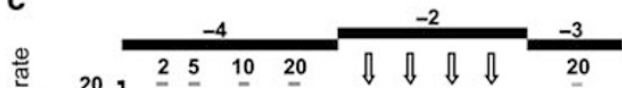

d

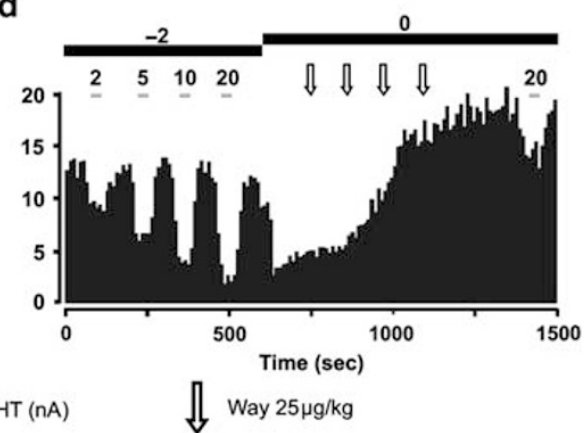

e

Tonic Activation
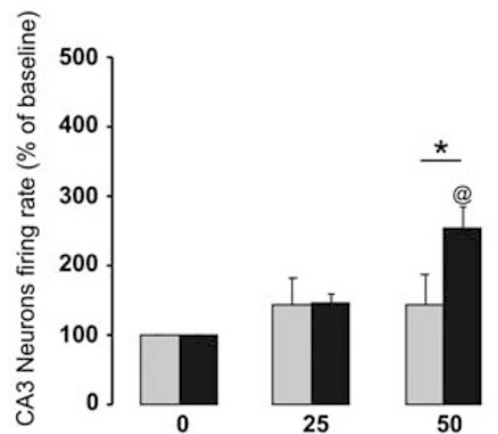

50

75

WAY $100635(\mu \mathrm{g} / \mathrm{kg})$

Figure 4 (a, b) Data are mean ( \pm SEM) of $R T_{50}$ values and $\mathrm{IT}_{50}$ index calculated from microiontophoretic applications of 5-HT in control ( $\left.\mathbf{\square}\right)$ and rats treated with agomelatine for 14 days ( $\mathbf{\square})$. (c, d) Integrated firing rate histograms of dorsal hippocampus CA3 pyramidal neurons illustrating intravenous administration of incremental doses of $25 \mu \mathrm{g} / \mathrm{kg}$ of WAY-100,635 in vehicle (a) and I4-day agomelatine (b) rats. (e) Effect of intravenous administration of incremental doses of the 5-HTIA antagonist WAY-I00,635 on basal firing rate in control ( $\mathbf{\square})$ and rats treated with agomelatine for 14 days ( $\mathbf{\square}$ ). *Significantly greater effect in agomelatine-treated rats in comparison with the control animals receiving the same dose of WAY-100,635, * $p<0.05$, *** $p<0.01$, **** $p<0.001$. ${ }^{@}$ Significant increase in the firing rate induced by WAY-I00,635 in treated rats ${ }^{9} p<0.05,{ }^{@ @ ~} p<0.01$, @@@ $p<0.001$.

LC-NE neurons, but no changes in the firing pattern of VTA-DA neurons (Millan et al, 2003). This is also in line with in vitro data showing that perfusion of DRN slices with agomelatine has no effect on the firing rate of DRN-5-HT neurons (Hanoun et al, 2004).

Interestingly, it was previously demonstrated that shortterm administration of the selective $5-\mathrm{HT}_{2 \mathrm{C}}$ receptor antagonist SB-242084 has no effect on the firing rate of LC-NE neurons (Dremencov et al, 2007b) and on the number of spontaneously active VTA-DA neurons (Chenu et al, 2009). Therefore, the short-term effect of agomelatine on both the LC and VTA neurons might be mediated, at least in part, by its melatonergic component. However, it was previously reported that an acute administration of melatonin $(16 \mathrm{mg} / \mathrm{kg})$ is devoid of effect on the firing rate of LC-NE neurons, whereas agomelatine $(4 \mathrm{mg} / \mathrm{kg})$ induces a significant increase (Millan et al, 2003). The effect on the LC-NE neuron may thus be due to the combination of the MT1/MT2 and 5- $\mathrm{HT}_{2 \mathrm{C}}$ activities of agomelatine. This is consistent with a single dose of the melatonergic receptor antagonist S22153 antagonizing the effect of short-term administration of agomelatine on the firing rate of LC-NE neurons (Figure 1e). Nevertheless, as agomelatine is also acting as an antagonist at $5-\mathrm{HT}_{2 \mathrm{~B}}$ receptors, it could not be excluded that this affinity may account for the enhancement of LC-NE activity. Indeed, even though the blockade of the $5-\mathrm{HT}_{2 \mathrm{C}}$ receptor has no effect by itself, it has been previously demonstrated that the $5-\mathrm{HT}_{2 \mathrm{~B} / 2 \mathrm{C}}$ receptor antagonist S32006 dose-dependently increases the firing rate of LC neurons (Dekeyne et al, 2008).

It is now well established that VTA-DA neurons receive a GABA input producing inhibitory postsynaptic potentials (IPSPs) that regulate the number of spontaneously active neurons in the VTA (Grace et al, 2007). It is therefore probable that the increase in the number of spontaneously active neurons (following short-term and long-term administration of agomelatine) was because of a disinhibition of VTA-DA neurons through a decrease of GABAinduced IPSPs. Similar increases of spontaneously active neurons have already been reported following chronic 


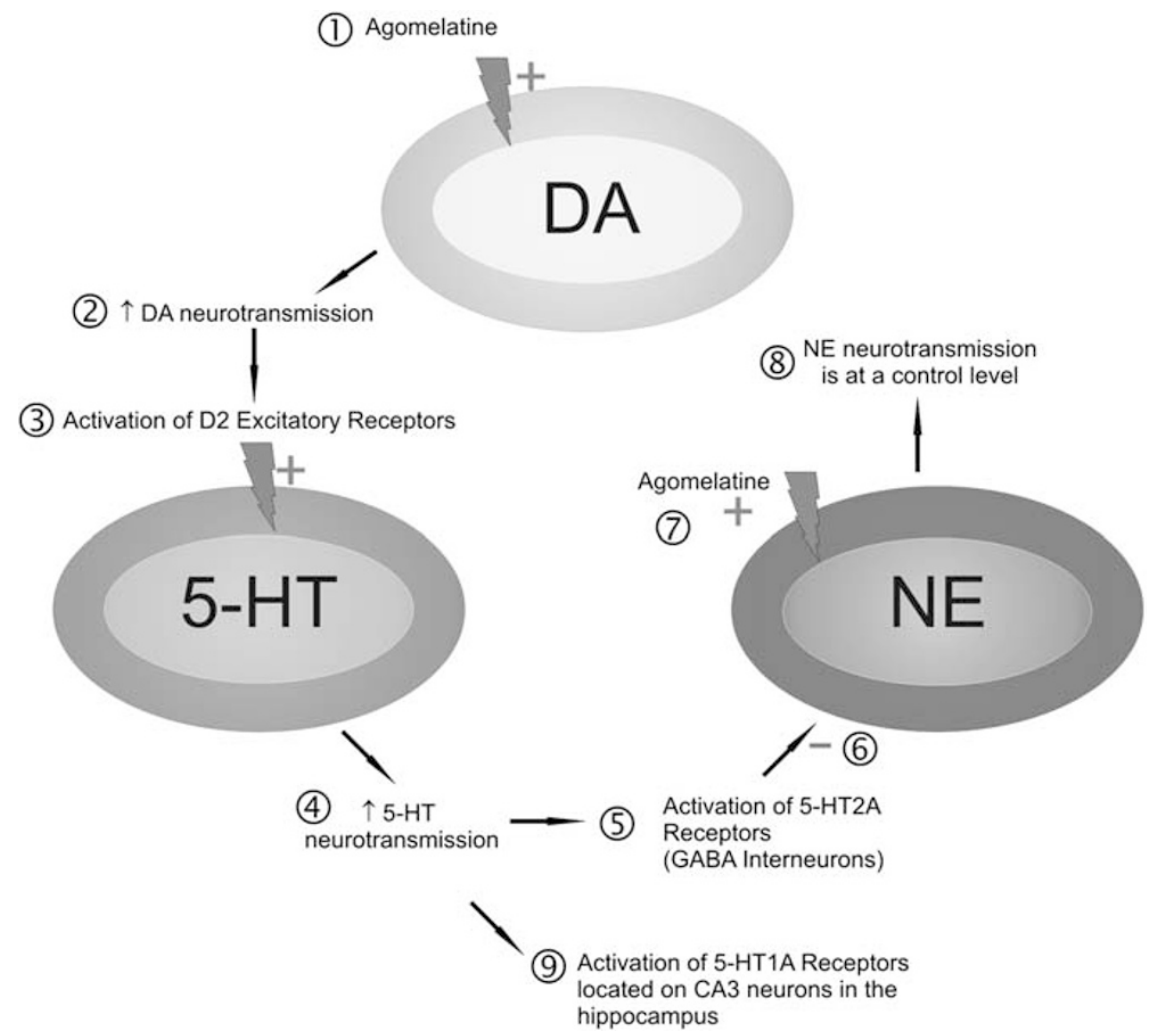

Figure 5 Long-term effect of agomelatine on monoaminergic systems. (1) Agomelatine ( 14 days) induced an excitatory effect on DA cells in the VTA (increase in the number of neuron spontaneously active and bursting activity) that resulted in (2) an increase in DA neurotransmission, leading to (3) the activation of excitatory D2 receptors located on DRN-5-HT cells. (4) The corresponding increase in 5-HT firing activity then induced (5) an activation of the excitatory 5- $\mathrm{HT}_{2}$ A receptors located on GABA interneurons. The increase in GABA activity induced (6) an inhibition of LC-NE neurons. However, (7) agomelatine by itself also exerts an excitatory effect on LC-NE neurons (as seen after a 2-day treatment). (8) Therefore, with the LC-NE neurons being under an excitatory and an inhibitory influence, the firing rate was in the range of that of the control animals. The increase in 5-HT firing activity (4)) also induced (9) a tonic activation of the inhibitory postsynaptic 5-HT IA receptors that may contribute to decrease the hyperactivity of the hippocampus commonly observed in patients suffering from MDD.

administration of various antidepressants such as citalopram, clorgyline, fluoxetine, and paroxetine (Chenu et al, 2009; Sekine et al, 2007).

The prolonged administration of agomelatine also induced a modification of the pattern of discharge of VTA-DA neurons. Indeed, even though the average firing rate of these neurons was unchanged, there was a significant increase in the bursting activity of these neurons (percent of spikes occurring in burst and number of bursts per min; Figure 2a). In a previous set of experiments, it was demonstrated that long-term administration of the selective $5-\mathrm{HT}_{2 \mathrm{C}}$ receptor antagonist SB-243213 induces an increase in the number of spikes per burst and in the percentage of neurons displaying bursts, but is also accompanied by a decrease in the number of bursts (Blackburn et al, 2002). This suggests that the $5-\mathrm{HT}_{2 \mathrm{C}}$ antagonistic properties of agomelatine cannot fully explain the modification of the firing pattern of VTA-DA neurons. Nonetheless, the same group subsequently reported that the $5-\mathrm{HT}_{2 \mathrm{~B} / 2 \mathrm{C}}$ receptors antagonist SB-200646A given chronically to rats at a high dose of $20 \mathrm{mg} / \mathrm{kg} /$ day (but not at a lower dose of $10 \mathrm{mg} / \mathrm{kg} /$ day) increases the number of spikes per burst, the percentage of spikes occurring in bursts and the number of bursts (Blackburn et al, 2006) as was found in the present study. Taken together, these data suggest that agomelatine might increase the bursting activity of VTA-DA neurons through a blockade of both $5-\mathrm{HT}_{2 \mathrm{~B}}$ and $5-\mathrm{HT}_{2 \mathrm{C}}$ receptors. However, in the present study a single administration of the melatonergic receptor antagonist S22153 reversed the increase in the number of bursts induced by a chronic regimen of agomelatine; therefore, the melatonergic component of agomelatine might also be involved in the modification of the pattern of discharge of VTA-DA neurons. The single injection of S22153 did not antagonize the increase in the percentage of spikes occurring in bursts. This is mainly because of the fact that it decreased both the number of bursts and the firing rate of VTA-DA neurons. The involvement of melatonin in the control of DA transmission is in line with data showing that a chronic treatment with melatonin alone induces an increase in the tissular level of DA in old rats (Esteban et al, 2010). Further experiments are necessary to determine whether melatonin by itself can induce a modification of the discharge pattern of VTA-DA neurons.

As the enhancement of the firing rate of DRN-5-HT neurons only appeared after the long-term regimen, it was thus likely that this was an indirect effect of agomelatine. Indeed, it has been repeatedly demonstrated that both the $\mathrm{NE}$ and the DA systems exert an excitatory effect on the DRN-5-HT neurons. These excitatory effects of NE and DA 
on DRN-5-HT neurons have been discussed in previous papers (Chernoloz et al, 2009, 2012; Ghanbari et al, 2010; Katz et al, 2010). Briefly, the excitatory effect of NE is mediated by the activation of $\alpha_{1}$-adrenoreceptors, whereas the excitatory effect of DA is mediated by the activation of the $\mathrm{D} 2$ receptors. As the electrical activity of LC-NE neuron was back to the control level after a 2-week repeated exposure to agomelatine, the enhancement of DRN-5-HT neuron was therefore likely a consequence of the increase of DA activity, similar to what is obtained after short- and longterm administration of the D2 receptor antagonist aripiprazole (Chernoloz et al, 2009). This was confirmed by the experiments showing that the blockade of the D2 receptors by a single administration of paliperidone, which is devoid of effect on its own, reversed the increase of firing rate of DRN5-HT neurons induced by agomelatine. Taken together with the results showing that a 2-day administration of agomelatine induced a $70 \%$ increase in the number of spontaneously active DA neurons without increasing the firing rate of 5-HT neurons, these data suggest that both parameters (bursts and number of active neurons) have to be increased to induce a tonic excitation of 5-HT neurons. This was also indirectly confirmed by the experiments showing that a single administration of the melatonergic receptor antagonist S22153 antagonized both the increase in DA and 5-HT transmission. This enhancement of 5-HT firing led to an enhancement of the tonic activation of $5-\mathrm{HT}_{1 \mathrm{~A}}$ receptors in hippocampus, as indicated by disinhibition of firing activity by WAY-100,635. Indeed, administration of the $5-\mathrm{HT}_{1 \mathrm{~A}}$ receptor antagonist WAY-100,635 increased the firing rate of $\mathrm{CA} 3$ neurons in rats given repeated injections of agomelatine for 14 days, but not in control rats.

A similar enhancement of 5-HT transmission in the LC may explain the reversal of the increased firing of LC-NE neurons after 2 weeks of agomelatine. Indeed, it has previously been demonstrated that the increased activation of $5-\mathrm{HT}_{2 \mathrm{~A}}$ receptors located on GABA interneurons turns down the firing rate of LC-NE neurons (Szabo and Blier, 2001). In studies using long-term exposure to selective serotonin reuptake inhibitors, the firing rate of LC-NE neurons is decreased, whereas in the case of agomelatine, the firing rate remains at the control level. This is mainly because of the fact that agomelatine exerts an excitatory effect on NE firing after an acute and two daily injections. Therefore, LC-NE neurons may be under an excitatory influence by agomelatine and an inhibitory one through 5HT via GABA, thereby resulting in a firing rate in the range of that of the control animals.

Taken together, these results explain how and why the administration of a single dose of the melatonergic receptor antagonist S22153 antagonized the antidepressant-like effect of agomelatine in animal models of depression. The present results suggest that (1) agomelatine exerts direct (2 days) and indirect (14 days) modulations of monoaminergic neuronal activity and that (2) the melatonergic agonistic activity of agomelatine contributes to the enhancement of $\mathrm{DA}$ and 5-HT neurotransmission (Figure 5). The most commonly used antidepressants, the selective serotonin reuptake inhibitors, induce an increase in 5-HT transmission that will in turn dampen the electrical activity of LC-NE neurons (Szabo and Blier, 2001) and VTA-DA neurons (Dremencov et al, 2009). However, although long-term administration of agomelatine induced no alteration of $\mathrm{NE}$ neurons, it increased DA and 5-HT transmission. These effects, together with the absence of monoamine uptake blockade properties of the drug, strengthen the differences between agomelatine and classical antidepressants.

\section{ACKNOWLEDGEMENTS}

This study was supported by a grant from IRIS (Institut de Recherches Internationales Servier). Dr Blier was in receipt of an Endowed Chair from the University of Ottawa Institute of Mental Health Research and the Canada Research Chair in Psychopharmacology.

\section{DISCLOSURE}

Dr El Mansari and Dr Chenu declare no conflict of interest. Dr Blier received grants and/or honoraria from Astra Zeneca, Bristol-Myers Squibb, Eli Lilly, Janssen, Labopharm, Lundbeck/Takeda, Merck, Pfizer, Servier, and Wyeth.

\section{REFERENCES}

Aghajanian GK, Vandermaelen CP (1982). Intracellular identification of central noradrenergic and serotonergic neurons by a new double labeling procedure. J Neurosci 2: 1786-1792.

Allers KA, Sharp T (2003). Neurochemical and anatomical identification of fast-and slow-firing neurones in the rat dorsal raphe nucleus using juxtacellular labelling methods in-vivo. Neuroscience 122: 193-204.

Aman TK, Shen RY, Haj-Dahmane S (2007). D2-like dopamine receptors depolarize dorsal raphe serotonin neurons through the activation of nonselective cationic conductance. J Pharmacol Exp Ther 320: 376-385.

Audinot V, Mailliet F, Lahaye-Brasseur C, Bonnaud A, Le Gall A, Amossé $C$ et al (2003). New selective ligands of human cloned melatonin MT1 and MT2 receptors. Naunyn Schmiedebergs Arch Pharmacol 367: 553-561.

Bertaina-Anglade V, la Rochelle CD, Boyer PA, Mocaer E (2006). Antidepressant-like effects of agomelatine (S 20098) in the learned helplessness model. Behav Pharmacol 17: 703-713.

Blackburn TP, Minabe Y, Middlemiss DN, Shirayama Y, Hashimoto K, Ashby Jr CR (2002). Effect of acute and chronic administration of the selective 5-HT2C receptor antagonist SB243213 on midbrain dopamine neurons in the rat: an in vivo extracellular single cell study. Synapse 46: 129-139.

Blackburn TP, Suzuki K, Ashby Jr CR (2006). The acute and chronic administration of the $5-\mathrm{HT}(2 \mathrm{~B} / 2 \mathrm{C})$ receptor antagonist SB-200646A significantly alters the activity of spontaneously active midbrain dopamine neurons in the rat: an in vivo extracellular single cell study. Synapse 59: 502-512.

Bourin M, Mocaer E, Porsolt R (2004). Antidepressant-like activity of S 20098 (agomelatine) in the forced swimming test in rodents: involvement of melatonin and serotonin receptors. J Psychiatry Neurosci 29: 126-133.

Chenu F, El Mansari M, Blier P (2009). Long-term administration of monoamine oxidase inhibitors alters the firing rate and pattern of dopamine neurons in the ventral tegmental area. Int $J$ Neuropsychopharmacol 12: 475-485.

Chernoloz O, El Mansari M, Blier P (2009). Electrophysiological studies in the rat brain on the basis for aripiprazole augmentation of antidepressants in major depressive disorder. Psychopharmacology (Berl) 206: 335-344.

Chernoloz O, El Mansari M, Blier P (2012). Long-term administration of the dopamine $\mathrm{D} 3 / 2$ receptor agonist pramipexole 
increases dopamine and serotonin neurotransmission in the male rat forebrain. J Psychiat Neurosci 37: 113-121.

Dalton EJ, Rotondi D, Levitan RD, Kennedy SH, Brown GM (2000). Use of slow-release melatonin in treatment-resistant depression. J Psychiat Neurosci 25: 48-52.

de Bodinat C, Guardiola-Lemaitre B, Mocaër E, Renard P, Muñoz C, Millan MJ (2010). Agomelatine, the first melatonergic antidepressant: discovery, characterization and development. Nat Rev Drug Discov 9: 628-642.

Dekeyne A, Mannoury la Cour C, Gobert A, Brocco M, Lejeune F, Serres F et al (2008). S32006, a novel 5-HT2C receptor antagonist displaying broad-based antidepressant and anxiolytic properties in rodent models. Psychopharmacology (Berl) 199: 549-568.

Dolberg OT, Hirschmann S, Grunhaus L (1998). Melatonin for the treatment of sleep disturbances in major depressive disorder. Am J Psychiatry 155: 1119-1121.

Dremencov E, El Mansari M, Blier P (2007a). Distinct electrophysiological effects of paliperidone and risperidone on the firing activity of rat serotonin and norepinephrine neurons. Psychopharmacology (Berl) 194: 63-72.

Dremencov E, El Mansari M, Blier P (2007b). Noradrenergic augmentation of escitalopram response by risperidone: electrophysiologic studies in the rat brain. Biol Psychiatry 61: 671-678.

Dremencov E, El Mansari M, Blier P (2009). Effects of sustained serotonin reuptake inhibition on the firing of dopamine neurons in the rat ventral tegmental area. J Psychiatry Neurosci 34: 223-229.

Esteban S, Garau C, Aparicio S, Moranta D, Barcelo P, Fiol MA et al (2010). Chronic melatonin treatment and its precursor Ltryptophan improve the monoaminergic neurotransmission and related behavior in the aged rat brain. J Pineal Res 48: 170-177.

Ghanbari R, El Mansari M, Blier P (2010). Electrophysiological effects of the co-administration of escitalopram and bupropion on rat serotonin and norepinephrine neurons. J Psychopharmacol 24: 39-50.

Gobert A, Rivet JM, Lejeune F, Newman-Tancredi A, AdhumeauAuclair A, Nicolas JP et al (2000). Serotonin(2C) receptors tonically suppress the activity of mesocortical dopaminergic and adrenergic, but not serotonergic, pathways: a combined dialysis and electrophysiological analysis in the rat. Synapse 36: 205-221.

Grace AA, Bunney BS (1983). Intracellular and extracellular electrophysiology of nigral dopaminergic neurons-1. Identification and characterization. Neuroscience 10: 301-315.

Grace AA, Bunney BS (1984). The control of firing pattern in nigral dopamine neurons: burst firing. J Neurosci 4: 2877-2890.

Grace AA, Floresco SB, Goto Y, Lodge DJ (2007). Regulation of firing of dopaminergic neurons and control of goal-directed behaviors. Trends Neurosci 30: 220-227.

Haddjeri N, Blier P, de Montigny C (1998). Long-term antidepressant treatments result in a tonic activation of forebrain 5-HT1A receptors. J Neurosci 18: 10150-10156.

Haj-Dahmane S (2001). D2-like dopamine receptor activation excites rat dorsal raphe 5-HT neurons in vitro. Eur J Neurosci 14: 125-134.

Hajos M, Gartside SE, Villa AE, Sharp T (1995). Evidence for a repetitive (burst) firing pattern in a sub-population of 5-hydroxytryptamine neurons in the dorsal and median raphe nuclei of the rat. Neuroscience 69: 189-197.

Hale A, Corral RM, Mencacci C, Ruiz JS, Severo CA, Gentil V (2010). Superior antidepressant efficacy results of agomelatine versus fluoxetine in severe MDD patients: a randomized, doubleblind study. Int Clin Psychopharmacol 25: 305-314.

Hanoun N, Mocaer E, Boyer PA, Hamon M, Lanfumey L (2004). Differential effects of the novel antidepressant agomelatine (S
20098) versus fluoxetine on 5-HT1A receptors in the rat brain. Neuropharmacology 47: 515-526.

Kandel ER, Spencer WA, Brinley Jr FJ (1961). Electrophysiology of hippocampal neurons. I. Sequential invasion and synaptic organization. J Neurophysiol 24: 225-242.

Kasper S, Hajak G, Wulff K, Hoogendijk WJ, Montejo AL, Smeraldi E et al (2010). Efficacy of the novel antidepressant agomelatine on the circadian rest-activity cycle and depressive and anxiety symptoms in patients with major depressive disorder: a randomized, doubleblind comparison with sertraline. J Clin Psychiatry 71: 109-120.

Kasper S, Hamon M (2009). Beyond the monoaminergic hypothesis: agomelatine, a new antidepressant with an innovative mechanism of action. World J Biol Psychiatry 10: 117-126.

Katz NS, Guiard BP, El Mansari M, Blier P (2010). Effects of acute and sustained administration of the catecholamine reuptake inhibitor nomifensine on the firing activity of monoaminergic neurons. J Psychopharmacol 24: 1223-1235.

Kennedy SH, Emsley R (2006). Placebo-controlled trial of agomelatine in the treatment of major depressive disorder. Eur Neuropsychopharmacol 16: 93-100.

Lemoine P, Guilleminault C, Alvarez E (2007). Improvement in subjective sleep in major depressive disorder with a novel antidepressant, agomelatine: randomized, double-blind comparison with venlafaxine. J Clin Psychiatry 68: 1723-1732.

Millan MJ, Brocco M, Gobert A, Dekeyne A (2005). Anxiolytic properties of agomelatine, an antidepressant with melatoninergic and serotonergic properties: role of 5-HT2C receptor blockade. Psychopharmacology (Berl) 177: 448-458.

Millan MJ, Gobert A, Lejeune F, Dekeyne A, Newman-Tancredi A, Pasteau $\mathrm{V}$ et al (2003). The novel melatonin agonist agomelatine (S20098) is an antagonist at 5-hydroxytryptamine2C receptors, blockade of which enhances the activity of frontocortical dopaminergic and adrenergic pathways. J Pharmacol Exp Ther 306: 954-964.

Papp M, Gruca P, Boyer PA, Mocaer E (2003). Effect of agomelatine in the chronic mild stress model of depression in the rat. Neuropsychopharmacology 28: 694-703.

Pineyro G, Blier P, Dennis T, de Montigny C (1994). Desensitization of the neuronal 5-HT carrier following its long-term blockade. J Neurosci 14: 3036-3047.

Racagni G, Riva MA, Molteni R, Musazzi L, Calabrese F, Popoli M et al (2011). Mode of action of agomelatine: synergy between melatonergic and 5-HT(2C) receptors. World J Biol Psychiatry 12: 574-587.

Sekine Y, Suzuki K, Ramachandran PV, Blackburn TP, Ashby Jr CR (2007). Acute and repeated administration of fluoxetine, citalopram, and paroxetine significantly alters the activity of midbrain dopamine neurons in rats: an in vivo electrophysiological study. Synapse 61: 72-77.

Szabo ST, Blier P (2001). Functional and pharmacological characterization of the modulatory role of serotonin on the firing activity of locus coeruleus norepinephrine neurons. Brain Res 922: 9-20.

Ungless MA, Magill PJ, Bolam JP (2004). Uniform inhibition of dopamine neurons in the ventral tegmental area by aversive stimuli. Science 303: 2040-2042.

Valenti O, Cifelli P, Gill KM, Grace AA (2011). Antipsychotic drugs rapidly induce dopamine neuron depolarization block in a developmental rat model of schizophrenia. J Neurosci 31: 12330-12338.

Ying SW, Rusak B, Delagrange P, Mocaer E, Renard P, GuardiolaLemaitre B (1996). Melatonin analogues as agonists and antagonists in the circadian system and other brain areas. Eur J Pharmacol 296: 33-42. 